Einführung in das Schwerpunktthema

\title{
Marktintegration, Umweltschutz und Harmonisierung
}

Von Ulrich Petschow

$\mathrm{D}$ er Integrationsprozeß in Europa läßt sich höchst unterschiedlich interpretieren. Mit Fritz Scharpf läßt sich jedoch feststellen, daß im Rahmen der Europäischen Union die „positive Integration“, also gemeinsame marktgestaltende und -korrigierende Politiken auf europäischer Ebene, weit hinter der ,,negativen Integration", nämlich dem Abbau von Handelshemmnissen zur Schaffung eines einheitlichen Marktes, zurückbleibt (1). Auf Grundlage der Römischen Verträge (1957) und der Einheitlichen Europäischen Akte (1987) konnten die Europäische Kommission und der Europäische Gerichtshof den gemeinsamen Markt auf immer mehr Sachgebiete ausweiten, ohne daß der Europäische Ministerrat dazu eine Entscheidung treffen mußte. Die Marktintegration wurde somit automatisch und ,politisch unreflektiert“ vorangetrieben. Hingegen hängt die positive Integration, deren Grundsätze oft nicht explizit durch den Vertrag fixiert sind, jeweils von Ministerratsentscheidungen ab und ist aufgrund der hohen Konsenserfordernisse kaum umsetzbar. Im Ergebnis wurden die nationalstaatliche politische Einbettung der Wirtschaft gelockert und entsprechende Steuerungsmöglichkeiten erschwert, ohne daß es auf einer höheren Ebene $\mathrm{zu}$ einer entsprechenden Reregulierung gekommen ist. Direkte Einschränkungen für die nationale Politik ergeben sich aus den rechtlichen Grundlagen des Marktes, also den vier Freiheiten (für Waren, Dienstleistungen, Kapital und Arbeit) und dem Wettbewerbsrecht, welche bestimmte Politikinstrumente ausschließen bzw. deren Anwendung schwieriger machen. Dadurch werden die Autonomiespielräume der Mitgliedsstaaten zunehmend eingeschränkt. Indirekte Autonomiebeschränkungen ergeben sich dadurch, daß sich durch die vier Freiheiten die mobilen Faktoren dem regulierenden Zugriff des Nationalstaates entziehen können.

Nichtsdestotrotz hat sich eine EU-Umweltpolitik als gemeinschaftliches Handlungsfeld entwickelt (vgl. Stichwort: Europäische Harmonisierung auf S. 13). Vielfach konnte durch materielle und prozedurale Vorgaben als Ergebnis des Harmonisierungsprozesses sogar eine Verbesserung der Umweltstandards erreicht werden (2).

Dieses Spannungsverhältnis zwischen Umweltpolitik und Marktintegration wurde im Rahmen eines EU-Forschungsprojektes mit dem Titel "SustainWaste“ unter Beteiligung von Wissenschaftlern aus fünf Ländern (Griechenland, Deutschland, Italien, Frankreich und Holland) an dem Beispiel (Haushalts-) Abfallpolitik vertieft untersucht. In den angeführten Ländern wurden die langfristigen Entwicklungslinien der nationalen Politiken sowie die Spannungen bzw. Interaktionen zwischen den Abfallregimen untersucht.

\section{Neve Sichtweise}

Als theoretische Ansätze dienten die Theorie der Konventionen und die Regimetheorie (vgl. den Beitrag von Godard in diesem Heft). Daraus folgt eine Erweiterung der Sichtweise auf Harmonisierungsprozesse innerhalb der EU, die die unterschiedlichen „Weltsichten“ in den jeweiligen Nationalstaaten zum Ausgangspunkt nimmt. Diese sind das Ergebnis von Konventionen, die in kollektiven Prozessen entstehen, um die allgegenwärtigen Unsicherheiten zu bewältigen. Der wirtschaftliche Imperativ des freien Binnenmarktes berücksichtigt solche kollektiven Prozesse nur sehr begrenzt und wenn, dann vorrangig als Hemmnis für die wirtschaftliche Liberalisierung. Gerade die unterschiedlichen Konventionen und Wertvorstellungen in den Ländern führen aber zu den wesentlichen Konflikten zwischen unterschiedlichen Regimen und werfen die Frage auf, welche Harmonisierungsschritte in welcher Form geeignet sein können, um die prinzipiellen Vorteile offener Märkte nicht in Frage zu stellen. Rein ökonomische effizienzorientierte Betrachtungen greifen hier zu kurz, gerade weil wesentliche Elemente der Entscheidungsfindung und Wertvorstellungen nicht einbezogen werden. Ein entscheidendes Element der europäischen Integration wäre es insofern, zu gemeinsamen Vorstellungen über die Entwicklung der EU und die Fragen des
Umweltschutzes und der Nachhaltigkeit zu kommen. Dies kann letztlich allein in einer prozeßorientierten Herangehensweise durch diskursive Prozesse auf vielen Ebenen der jeweiligen Nationalstaaten erfolgen. Harmonisierungsprozesse könnten in der Folge wesentlich friktionsloser etabliert werden.

Eine wesentliche Rolle können in diesem Zusammenhang eine Orientierung der Politik am Ziel der Nachhaltigkeit, ,best practice“-Beispiele und die Dissemination von Erfahrungen spielen. Dies setzt aber voraus, daß solche Erfahrungen und Lernprozesse im Umgang mit Umweltproblemen auf den jeweiligen nationalen Ebenen gemacht werden können. Es sollte eine Konkurrenz um die „besten“ ökonomisch-ökologischen Lösungen stattfinden. Insofern ist es nicht allein die EUEbene, die Harmonisierungsprozesse von „oben “ anstoßen sollte, sondern Anstöße in diese Richtung sollten gerade auch von ,unten“, d.h. den jeweiligen Umweltpolitiken und Akteuren kommen. Es geht damit mehr um Prozesse in Richtung einer Nachhaltigen Entwicklung, die als Ergebnis die Harmonisierung zur Folge haben, als um die Durchsetzung von Harmonisierungsvorgaben, die unterschiedliche Startpunkte nicht hinreichend berïcksichtigen.

Die Analyse der Abfallpolitik verdeutlicht die angedeuteten Probleme von Harmonisierungsprozessen exemplarisch. Im Grundsatz weisen die nationalen Abfallpolitiken ähnliche Entwicklungstendenzen auf; diese laufen bzw. liefen aber nicht zeitlich synchron ab und unterscheiden sich zudem in den zugrundeliegenden Konventionen und Prinzipien. Die ,prevention ladder" (Vermeidung vor Verwertung vor Beseitigung), die als Leitidee Anfang der 70er Jahre entwickelt wurde, wirkte mit einem erheblichen „time-lag“ auf die Abfallpolitik und war prägend z.B. für die Verpackungsverordnung, also die Verlagerung der Verantwortung für den Abfall auf die Hersteller und nicht mehr auf die entsorgungspflichtigen Körperschaften.

Diese abfallwirtschaftliche Umorientierung ist aber zugleich der wesentliche Grund dafuir, daß Friktionen zwischen den jeweiligen Regimes der unterschiedlichen Staaten auftreten. Die klassischen Prinzipien der Abfallwirtschaft wie das Autarkieprinzip, das Prinzip nahräumlicher Entsorgung etc. verlieren bei den nun zentralen Fragen der Wiederverwertung und des Recycling an Bedeutung. Das Management der Stoffströme überschreitet oft nationale Grenzen; nationalstaatliche Politiken induzieren häufig Handel oder 
D ie Harmonisierung von nationalen Politien ist ein Problemfeld, das die Europäische Union (EU) bzw. im wirtschaftlichen Zusammenhang die Europäische Gemeinschaft seit ihrer Gründung 1957 begleitet. Dabei sind aus ökologischer Perspektive zwei Dimensionen bedeutsam, deren Konflikt für einen Großteil der bestehenden Probleme verantwortlich ist:

- Die handelspolitisch motivierte Harmonisierung von (nicht nur) umweltpolitischen Regulierungen und

- die Entwicklung einer gemeinsamen europäischen Umweltpolitik.

Diese Zweiteilung spiegelt sich auch im EG-Vertrag wider. Im Kern der Europäischen Integration steht die Bildung eines gemeinsamen Binnenmarkts, d.h. der vertraglich festgelegte Abbau aller Handelshemmnisse (Art. 100 des EGVertrags, Art. 94 in der neuen Nomenklatur des Amsterdamer Vertrages von 1997). Als solche kommen potentiell auch Umweltregulierungen in Frage. Lange Zeit galt die Priorität der Handels- vor der Umweltpolitik und das Damoklesschwert des Art. 100 schwebte über jeder handelswirksamen nationalen Umweltmaßnahme.

aber wirken protektionistisch und haben hierdurch direkte Rückwirkungen auf die Nachbarländer zur Folge. Dies ist wiederum ein Antrieb für Versuche, die Abfallpolitik zu harmonisieren (3).

\section{Die Beiträge im einzelnen}

Die Beiträge des Schwerpunkts nehmen das Beispiel der Abfallpolitik zum Ausgangspunkt, um europäische Harmonisierungsprozesse und ihre Probleme aus verschiedenen Perspektiven zu beleuchten. Dabei steht weniger der konkrete Ablauf auf der europäischen Ebene im Mittelpunkt; vielmehr dienen die nationalen Entwicklungen und ihre Wechselwirkungen als Ausgangspunkt für die Analyse des Spannungsfelds zwischen europäischen und nationalen Regulierungen.

Der grundlegende Beitrag von Olivier Godard (Frankreich) entwickelt den oben nur angedeuteten konzeptionellen Rahmen für die Analyse von Harmonisierungsprozessen ausführlicher. Wim Hafkamp (Niederlande) zeichnet dann die Entwicklung des europäischen Abfallregimes insgesamt und für die einzelnen Länder nach. Er zeigt u.a., daß die Differenzen der Abfallpolitiken zwar groß sind, aber die unterschiedlichen Dynamiken zu Anpassungsreaktionen der Regime führen. Harmonisierung ist somit weniger ein Problem des „Moments“ denn des Prozesses.

Der Franzose Nicolas Buclet thematisiert am Beispiel der Verpackungspolitik in Dänemark und

\section{Stichwort: Europäische Harmonisierung}

Eine eigenständige Umweltpolitik betreibt die EU faktisch auf Initiative der Europäischen Kommission und mit Duldung durch den Europäischen Gerichtshof seit Mitte der 70 er Jahre. Eine formelle Zuständigkeit besitzt sie jedoch erst seit der Einheitlichen Europäischen Akte von 1986, wobei die Verantwortung für die Umsetzung gemäß dem Subsidiaritötsprinzip ouf der nationalen Ebene verbleibt. Es wurde einerseits ein Kapitel "Umwelt" (Art. 130r-t; heute Art. 174-176) in den EG-Vertrag eingefügt, unter dos olle nicht direkt binnenmarktrelevanten Regelungen fallen. Hier wird den Mitgliedsstaaten prinzipiell eine nationale Vorreiterrolle zugestanden. Andererseits wurde das Binnenmarktkapitel in Art. 100a,b (heute Art. 95) umweltpolitisch ergönzt und auch hier ein "hohes Schutzniveau" garantiert, aber auch die gegenseitige Akzeptanz nationaler Standards festgeschrieben. (Neve) nationale

Deutschland das Spannungsverhältnis zwischen den grundsätzlichen Zielen des europäischen Binnenmarktes und ökologisch begründeten nationalen abfallpolitischen Maßnahmen. Er kommt zu dem Ergebnis, daß wesentliche Maßnahmen sehr viel stärker mit den Interessenlagen einzelner Industriezweige zu erklären sind, als mit dem angegebenen Motiv des Umweltschutzes.

Lilo Fischer widmet sich der Frage, inwieweit eine zunehmende Harmonisierung und die resultierende Beschränkung der Handlungsmöglichkeiten positive Dynamiken in Frage stellt, die durch nationale Vorreiter erzeugt werden könnten.

Antonio Massarutto (Italien) verdeutlicht schließlich, daß die Politiken der Mitgliedsländer vielfach nur bei oberflächlicher Betrachtung einander ähneln. Er betont, daß Harmonisierungsprozesse, die eine ,top down“-Strategie verfolgen, vielfach nur begrenzt erfolgreich sind bzw. sein können, wenn nicht die Implementationsbedingungen gerade auch in den Südländern der EU mit berücksichtigt werden.

Die Beiträge folgen damit nicht der ,gefestigten“ Sichtweise deutscher umweltpolitischer Überzeugungen, sondern versammeln z.T. durchaus kontroverse Ansichten von Wissenschaftlern aus europäischen Ländern mit unterschiedlichen umweltpolitischen Traditionen. Diese Kontrover-
Alleingänge bei produktbezogenen Umweltstandards sind jedoch seit Amsterdam nur noch möglich, wenn neue wissenschaftliche Erkenntnisse nachgewiesen oder spezifische Länderprobleme geltend gemocht werden.

Während der Beschlubfassungsprozeß innerhalb der europöischen Institutionen immer komplizierter wird, wurden im letzlich immer noch entscheidenden Ministerrat gemeinsame Umweltpolitiken Schritt für Schritt erleichtert. Seit dem Vertrag von Maastricht 1992 reicht - wie bei direkt binnenmarktrelevanten Regulierungen von jeher - eine qualifizierte Mehrheit für die Harmonisierung von Politiken aus. Ein Beispiel bildet die Altautorichtlinie, die Mitte Juli letzlich auch gegen den Willen Deutschlands verabschiedet werden konnte, nachdem Spanien und Großbritannien, die bis dato das Erreichen der notwendigen Stimmenzahl blockiert hatten, zustimmten. Einige ökologisch gewichtige Themenfelder unterliegen jedoch weiter dem Einstimmigkeitsprinzip; Beispiele bieten die Energieversorgung und alle Steverfragen und damit auch die Ökosteuer.

sen sind es jedoch, die die Entwicklung und Perspektiven der EU-Umweltpolitik prägen und prägen werden.

\section{Anmerkungen}

(1) Vgl. dazu Scharpf, F.W.: Balancing Positive and Negative Integration: The Regulatory Options for Europe. MPIfG Discussion Paper 1997/8;

Genschel, P.: Markt und Staat in Europo. In: Politische Vierteljahresschrift 39(1), 1998.

(2) Vgl. Héritier, A. et al.: Ringing the Changes in Europe. Regulatory Competition and the Transformation of the State. Berlin 1996;

Knill, C.: Liberalisierung und Umweltschutz. In: Ökologisches Wirtschaften 2/1998, S. 13-15.

(3) Als ein weiteres Ergebnis des Forschungsprojektes wurden Szenarien (von strikter Subsidiarität bis zu rodikaler Liberalisierung) entwickelt und einer Stärken-Schwächen-Analyse unterzogen. Die Projektergebnisse werden demnächst veröffentlicht, vgl. Buclet N., 0. Godard (eds.): Municipal Waste Management in Europe - A Comparative Study in Regime-building. Kluwer Academics Publ., Dordrecht \& London 1999.

\section{Der Autor}

Ulrich Petschow ist Leiter des Forschungsfelds Umweltökonomie und -politik am Institut für ökologische Wirtschaftsforschung.

Kontakt: I0̈W, Potsdamer Str. 105, 10785 Berlin. Tel. 030/884594-0, Fax 030/ 8825439,

E-mail: Ulrich.Petschow@ioew.de 
(c) 20I0 Authors; licensee IÖW and oekom verlag. This is an article distributed under the terms of the Creative Commons Attribution Non-Commercial No Derivates License (http://creativecommons.org/licenses/by-nc-nd/3.o/), which permits unrestricted use, distribution, and reproduction in any medium, provided the original work is properly cited. 